\title{
Preparative isolation of monomer catechins and oligomer procyanidin fractions from grape seed extracts by high-speed counter-current chromatography
}

\author{
Zhang Shuting ${ }^{1}$, Cui Yan $^{1}$, and Sun Baoshan ${ }^{1,2}$ \\ ${ }^{1}$ Shenyang Pharmaceutical University, No 103, Wenhua Road, 110016 Shenyang, P.R. China \\ ${ }^{2}$ Pólo Dois Portos, Instituto Nacional de Investigação Agrária e Veterinária, I.P., Quinta da Almoinha, 2565 - 191 Dois \\ Portos, Portugal
}

\begin{abstract}
In this paper, monomer (+)-catechin; (-)-epicatechin and (-)-epicatechin-3-o-gallate, three oligomeric procyanidin fractions (OPCs) and one polymeric procyanidin fractions (PPCs) were successfully separated and isolated from grape seed extracts using semi-preparative TBE-300 B high-speed counter-current chromatography (HSCCC). For their separation in monomer (+)-catechin; (-)-epicatechin and (-)-epicatechin 3O-gallate, hexane/ethyl acetate/water (1:10: 10, v/v/v) was selected as solvent system. For separation of OPCs and PPCs, solvent system ethyl acetate/water $(1: 1, \mathrm{v} / \mathrm{v})$ was selected. Compositional data and mean polymerization degrees of OPCs were determined by phloroglucinolysis method. The purity of each fraction obtained; monomer $(+)$-catechin (C), (-)-epicatechin (EC) and (-)-epicatechin-3-o-gallate (ECG), verified by UPLC, was 98\%, 82\% and $71 \%$, respectively. The mean polymerization degrees of the three OPCs fractions were respectively 2.842 , $3.521,4.475$. As compared with traditional separation methods for catechins and OPCs, HSCCC has shown to be a powerful and efficient technique for large-scale isolation of these compounds.
\end{abstract}

\section{Introduction}

Catechins and oligomeric proanthocyanidins (OPCs) are the most important phenolic compounds in grape and wine due to their extreme potency as antioxidants in the body. Scientific research has shown that these compounds are likely the most powerful antioxidants. Furthermore, Catechins and OPCs have active effects against both fat soluble and water soluble oxidizing agents in the body, therefore they are seen as being the most beneficial antioxidants in the natural world. Catechins and OPCs have proved to have biological activities such as protection of cardiovascular disease and the oxygen free radical scavenger capacity, anticancer, antitumor, anti-allergy, improving diabetic conditions, etc. [1-5].

Grape seed, a by-product of winemaking, is very rich in catechins and proanthocyanidins. The separation and preparation of catechins and OPCs from grape seeds are consequently of great market potential. Traditional separation methods for separation and preparation of catechins and OPCs are thin layer chromatography [6] (TLC), preparative liquid chromatography and column chromatography (CC) packed with silica gel, polyamide, macroporousresin or C18 [7-9]. But there are some disadvantages of these present methods, such as secondary pollution, complex process, long separation period, lowyield and high-cost.
Compared with traditional separation methods, highspeed counter-current chromatography (HSCCC) is featured with rapid separation, no samples adsorption, good repeatability and low cost. Without stationary phase as solid support, HSCCC can avoid high requirement of sample pretreatment resulting from solid carrier, as well as sample loss and sample denaturation caused by irreversible adsorption, which make separation more efficient $[3,10]$. HSCCC has been widely used in preparation and separation of natural products. N.S. Kumar et al. [11] separated seven catechins from the catechin extracts using high-speed counter-current chromatography. EGCG, EGC, GCG, ECG from black tea were separated by K.B. Wang et al. [3] using high-speed counter-current chromatography and G.B. Xia et al. [12] prepared and separated rare catechins by tannase-mediated biotransformation. But the grape seed crude extracts were unsuccessfully separated according to the polymerizaiton degree by HSCCC. And the separation and preparation of $\mathrm{C}, \mathrm{EC}$ and EGC from grape seed crude extracts was rarely attempted with a single HSCCC run.

The objective of this work was to isolate a preparative scale of catechins and OPCs from grape seed crude extract using HSCCC. By optimizing the solvent systems and separation conditions of HSCCC, C, EC and EGC were successfully separated from the grape seed crude extract. As well as OPCs and PPCs were obtained from PAs fraction. The developed HSCCC method of one 
Table 1. Two phase solvent systems and partition coefficients $(K)$ of C, EC and ECG.

\begin{tabular}{llllllll}
\hline \multirow{2}{*}{ Compound } & \multicolumn{3}{l}{ Hexane/ethyl acetate/methanol/water } & & \multicolumn{3}{c}{ Hexane/ethyl acetate/water } \\
\cline { 2 - 4 } \cline { 6 - 8 } & $1: 5: 1: 5$ & $1: 7: 1: 7$ & $1: 10: 1: 10$ & & $1: 5: 10$ & $1: 8: 10$ & $1: 10: 10$ \\
\hline $\mathrm{C}$ & 0.986 & 1.400 & 1.950 & & 1.054 & 1.780 & 2.253 \\
EC & 0.791 & 1.081 & 1.348 & & 0.799 & 1.210 & 1.479 \\
ECG & 2.474 & 4.761 & 5.744 & & 4.696 & 5.656 & 6.240 \\
\hline
\end{tabular}

step separation is more cost-efficient, providing a simple separation method for a large scale yields.

\section{Experimental}

\subsection{Reagents and materials}

Grape Seed Extracts (proanthocyanidins 95\%) were purchased from Tianjin JianFeng Natural Product R\&D Co., Ltd. (Chendu, China). (+)-Catechin (C, $\geq 98 \%)$, (-)epicatechin (EC, $\geq 98 \%$ ), (-)-epicatechin-3-o-gallate (ECG, $\geq 98 \%$ ) were purchased from Chendu Must BioTechnology Co., Ltd. (Tianjing, China). Phloroglucinolysis was purchased from Aladdin reagent (Shanghai, China). All organic solvents used for HSCCC and UPLC were purchased from Shandong Yuwang industrial Co., Ltd. Chemical branch (Shandong, China).

\subsection{Separation of catechins}

\subsubsection{Solvent system for HSCCC}

The solvent system has the primary role in HSCCC separation, while the partition coefficient $(\mathrm{K})$ values provide a good reference for the solvent system. According to references [11-17] and the preliminary results, the solvent system was adjusted on the basis of hexane/ethyl acetate/methanol/water and hexane/ethyl acetate/water. The K-values were determined by uv-spectrophotometer [18]. An amount of sample was added to a $10 \mathrm{ml}$ test tube with $4 \mathrm{ml}$ of each phase of the two phase solvent system. The test tube was shaken violently to reach equilibrium. The upper and lower phases were determined at $280 \mathrm{~nm}$. The $\mathrm{K}$-values were calculated according to the following formula: $\mathrm{K}=\mathrm{As} / \mathrm{Am}$ [18]. The K-values of six selected solvent systems were presented in Table 1 .

\subsubsection{HSCCC separation}

Monomer catechins were separated and isolated with hexane/ethyl acetate/water (1:10:10, v/v/v) as solvent system by a TBE-300 B HSCCC (Tauto Biotech, Shanghai, China). The coiled column was first filled with the upper organic phase as the stationary phase at a flow speed of $35 \mathrm{ml} / \mathrm{min}$. Then the apparatus was adjusted to a revolution speed of $900 \mathrm{rpm}$ while the lower aqueous phase as the mobile phase was pumped into the column at a flow speed of $2.0 \mathrm{~mL} / \mathrm{min}$. After the equilibrium, $20 \mathrm{ml}$ of the lower phase with $300 \mathrm{mg}$ of PAs were injected. The two elution modes were applied to catechins separation: $\mathrm{C}$ and $\mathrm{EC}$ were first eluted in the head-tail mode with the upper organic phase as the sationary phase and the lower aqueous phase as the mobile phase; after the head-tail mode for 300 min, ECG was eluted in the tail-head mode with the lower aqueous phase as the stationary phase and the upper organic phase as the mobile phase. The effluents were monitored with UV detector at $280 \mathrm{~nm}$. The fractions of the separated individual catechins were lyophilized and analyzed by UPLC.

\subsubsection{UPLC analysis}

The ACQUITY H-Class system used in this work is comprised of a Quaternary Solvent Manager (QSM), a Sample Manager with Flow through Needle(SM-FTN), and a Photodiode Array (PDA) Detector coupled to a data processing computer (Empower ${ }^{\mathrm{TM}} 2$ chromatography data software). The detection ranged from 200 to $500 \mathrm{~nm}$, being $280 \mathrm{~nm}$ for detection of individual catechins. The column was a ACQUITY UPLC BEH C18 $(50 \times 2.1 \mathrm{~mm}, 1.7 \mu \mathrm{m})$ (Waters) and the temperature was set at $30^{\circ} \mathrm{C}$. The flow rate of the mobile phase was fixed at $0.5 \mathrm{~mL} / \mathrm{min}$. Two elution solvents A (water: formic acid; 99.8:0.2, $\mathrm{v} / \mathrm{v}$ ) and B (acetonitrile: formic acid; 99.8:0.2, v/v) were used with the gradient elution program: $0 \mathrm{~min}$ (A98\%:B2\%), 1 min (A96\%:B4\%), 2 min (A88\%:B12\%), 4 min (A88\%:B12\%), 8 min (A68\%:B32\%), 9 min (A0\%: B100\%), 12 min (A98\%:B2\%) [19,20]. Individual catechins were confirmed by reference substance. The purity of the compounds was determined by area normalization method.

\subsection{Separation of PAs}

\subsubsection{Column chromatography separation}

PAs were fractionated by column chromatography on YMC ODS-A-HG to isolate monomer catechins, OPCs and PPCs as reported in our previous works [7-9]. Briefly, the aqueous extracts were loaded on the column pre-conditioned at distilled water. After washing with phosphate buffer $\mathrm{pH} 7.0$ to eliminate phenolic acids, the elution was first performed with $150 \mathrm{~mL}$ of ethyl acetate to elute catechins and OPCs, followed by $100 \mathrm{ml}$ methanol to elute PPCs fraction. For isolating catechins and OPCs fractions, the procedure was similar to be loaded on the column, elution was performed with $150 \mathrm{~mL}$ of ether to elute catechins, followed by $100 \mathrm{ml}$ methanol to elute OPCs fraction. The fractions will be evaporated to dryness under vacuum at $<30^{\circ} \mathrm{C}$, dissolved in distilled water and lyophilized.

\subsubsection{HSCCC separation}

On the Basis of the separation by column chromatography, PAs were fractioned with the solvent system comprising of ethyl acetate/water $(1: 1, \mathrm{v} / \mathrm{v})$, the rotation speed of $900 \mathrm{rpm}$, the flow speed of the mobile phase of $3 \mathrm{ml} / \mathrm{min}$, 

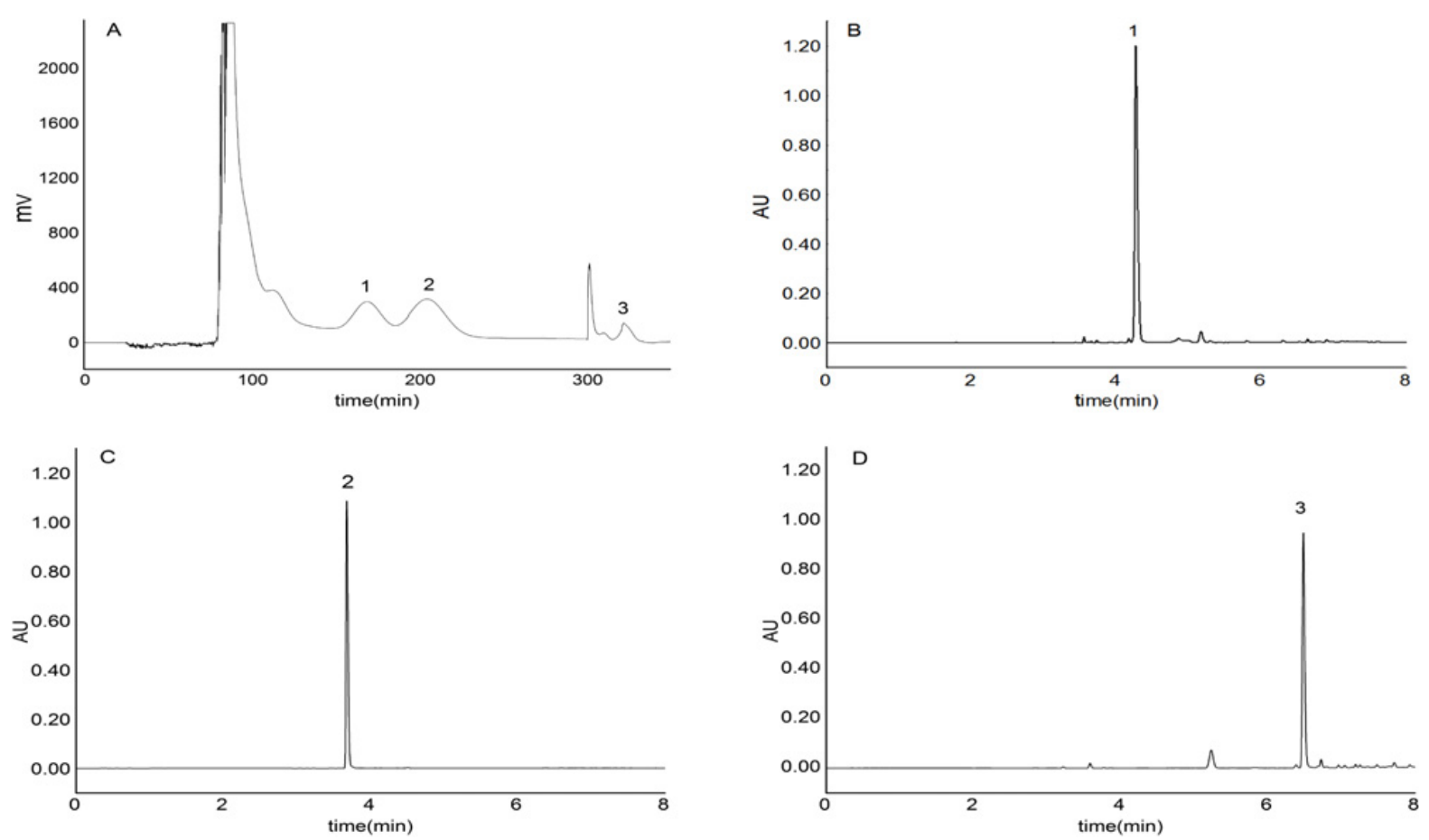

Figure 1. HSCCC separation of catechins (A) and UPLC analysis of fractions from HSCCC separation (B), (C), (D):1 (-)-epicatechin, 2 (+)-catechin, 3 (-)-Epicatechin-o-3-gallate.

the detection wavelength of $280 \mathrm{~nm}$, the temperature of $25^{\circ} \mathrm{C}$ and the injection volume of $20 \mathrm{ml}$ with $300 \mathrm{mg}$ of PAs. The two elution modes were applied to PAs separation: catechins and OPCs were first eluted in the tailhead mode with the lower aqueous phase as the stationary phase and the upper organic phase as the mobile phase; after the tail-head mode for $300 \mathrm{~min}$, PPCs were eluted in the head-tail mode with the upper organic phase as the stationay phase and the lower aqueous phase as the mobile phase. The fractions of the separated PAs were lyophilized.

\subsubsection{Hydrolysis of phloroglucinolysis}

The hydrolysis of phloroglucinolysis [21,22] in this work was applied to estimating the degree of polymerization and elucidating structural composition of proanthocyanidins. $1 \mathrm{ml}$ of PAs extracts in methanol $(6 \mathrm{mg} / \mathrm{ml})$ was added to $1 \mathrm{~mL}$ of phloroglucinol $(50 \mathrm{mg} / \mathrm{ml})$ in methanol acidified by $0.2 \mathrm{M} \mathrm{HCl}$ in a stoppered test tube. The tube was incubated for $20 \mathrm{~min}$ at $50^{\circ} \mathrm{C}$ for the complete phloroglucinolysis reaction and then put in cooling water to stop the reaction. The reaction mixture was filtered through $0.22 \mu \mathrm{m}$ filter and analyzed by UPLC. The UPLC conditions were the same as described above.

\section{Results and discussion}

\subsection{HSCCC separation of catechins}

A suitable solvent system is vital for a successful HSCCC separation. The properties of viscosity, polarity and density of different solvent system are far from different. The dissolution and distribution capacities of the same component are not the same in different solvent system. The solvent system is selected mainly basing on the main reference of the partition coefficient $(\mathrm{K})$ values and the polarity of the target components. While the $\mathrm{K}$-value is between 0.5 and 5, the separation is best. When the $\mathrm{K}$-value is too high or too low, the separation of the target components becomes difficult. As the K-value is too high, the components could be retained in the stationary phase and could not be flown out all the time. In addition, stationary phase retention rate above of being $30 \%$ can achieve better separation. In this paper, several solvent systems were attempted and quickly selected by the analytical HSCCC: with the upper organic phase of high polarity composed of n-butanol-ethyl acetate-water [23], the catechins were difficult to be eluted; while with the lower aqueous phase of high polarity composed of ethyl acetate/methanol/water $[11,12,24]$, the catechins were too quickly eluted to achieve separation; as the solvent system comprising of chloroform-methanol-water was concerned, it was too toxic to be applied in the field of food. According to the results of analytical HSCCC and the K-values, the two-phase solvent system was adjusted and determined on the basis of hexane/ethyl acetate/methanol/water [25] and hexane/ethyl acetate/water [26]. The separation was better as the retention rate was above $50 \%$ with the solvent system composed of hexane/ethyl acetate/methanol/water and hexane/ethyl acetate/water. It was shown in Table 1 that the smaller the ratio of hexane and methanol in the solvent system, the more the $\mathrm{K}$-values and the better they were separated; the $\mathrm{K}$-values ratio of catechin and epicatechin was higher in the solvent system composed of hexane/ethyl acetate/water (1:10:10, v/v/v), which made the best separation.

In one elution mode, the three catechins could not be simultaneously separated. The two elution mode was performed: the lower aqueous phase as the mobile phase 
Table 2. The mean degree of polymerizaiton of oligomeric proanthocyanidins (mmol).

\begin{tabular}{cccccccc}
\hline \multirow{2}{*}{ Samples } & \multicolumn{3}{c}{ Terminal units } & \multicolumn{3}{c}{ Extension units } & \multirow{2}{*}{ mDP } \\
\cline { 2 - 7 } & (+)-cat & $(-)$-epicat & $(-)$-epicatG & (+)-cat-p & $(-)$-epicat-p & (-)-epicatG-p & \\
\hline II & 1.076 & 1.325 & 0.229 & 1.086 & 3.323 & 0.436 & 2.842 \\
III & 1.255 & 0.490 & 0.175 & 0.439 & 3.954 & 0.447 & 3.521 \\
IV & 0.577 & 0.426 & 0.2053 & 0.513 & 3.096 & 0592 & 4.475 \\
\hline
\end{tabular}

was firstly in the head-tail mode, and then the upper organic phase as the mobile phase was in the tailhead mode. In addition, the flow speed also affected the separation. The lower the flow speed, the greater the retention rate of the stationary phase became with better separation. The optimal flow speed of TBE-300 HSCCC was $3 \mathrm{ml} / \mathrm{min}$, but ECG and its former impurity peek could not be separated. At the flow speed of $2 \mathrm{ml} / \mathrm{min}$, ECG could get higher retention rate to achieve better separation. And at the flow speed of $2 \mathrm{ml} / \mathrm{min}$, the longer the operation time in the head-tail mode lasted, the better ECG was separated. Finally the tail-head mode was applied at the flow speed of $2 \mathrm{ml} / \mathrm{min}$ after the head-tail mode for $300 \mathrm{~min}$, as shown in the Fig. 1.

At the temperature of $25^{\circ} \mathrm{C}$ or $30^{\circ} \mathrm{C}$, there was no great change in the stationary retention rate. As PAs were heat unstable, the higher tempature could not be applied. Thus, the temperature was set at $25^{\circ} \mathrm{C}$. The sample quantity was too large and the loss of the sationary phase was increasing. While the sample quanlity was too small, a large scale of separation and preparation could not be achieved. Hence, the sample quantity was fixed at $300 \mathrm{mg}$.

$\mathrm{C}$, EC and ECG were verified by their reference standard. As shown in the Fig. 1, their purity was $98 \%$, $82 \%, 71 \%$ by area normalization method.

\subsection{HSCCC separation of PAs}

In the current study, the column chromatography is mainly applied to the separation of PAs according to the polymerization degree [6-9]. But the method is complex process, consuming-time, low-yield and highcost. HSCCC can just overcome above disadvantages to separate PAs. Apple procyanidins were separated into different degrees of polymerization by HSCCC [27]. In this paper, On the basis of PAs separation by the column chromatography, polymeric proanthocyanidins could not be eluted with ethyl acetate as the mobile phase. The solvent system composed of ethyl acetate/water (1:1, $\mathrm{v} / \mathrm{v})$ was used to separate PAs according to the degree of polymerization. PAs fractions I, II, III and IV were eluted from the column. As it was shown in the Figs. 2 and 3 , the fraction I was only the monomer catechins, which was also analyzed by UPLC. The fraction I was verified by the reference standards, including (-)epicatechin, (+)-catechin and (-)-Epicatechin-3-o-gallate. The fraction II, III and IV were eluted from the column in increasing order of their polymerization degree. The fraction II, III and IV as OPCs were collected and analyzed by UPLC. As shown in table 2, their polymerization degree was respectively $2.842,3.521$, and 4.475 .

In the separation of PAs according to their degree of polymerization, the two elution modes were applied: the

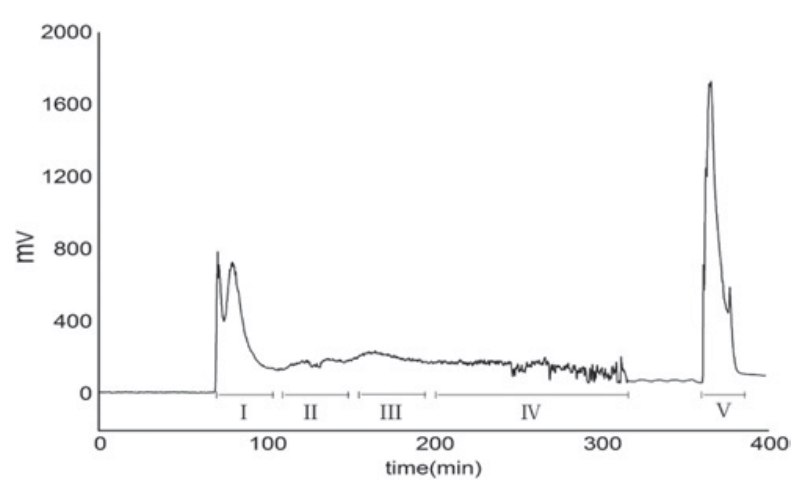

Figure 2. HSCCC separation of PAs: I as catechins, II, III and IV as $\mathrm{OPC}, \mathrm{V}$ as PPC.

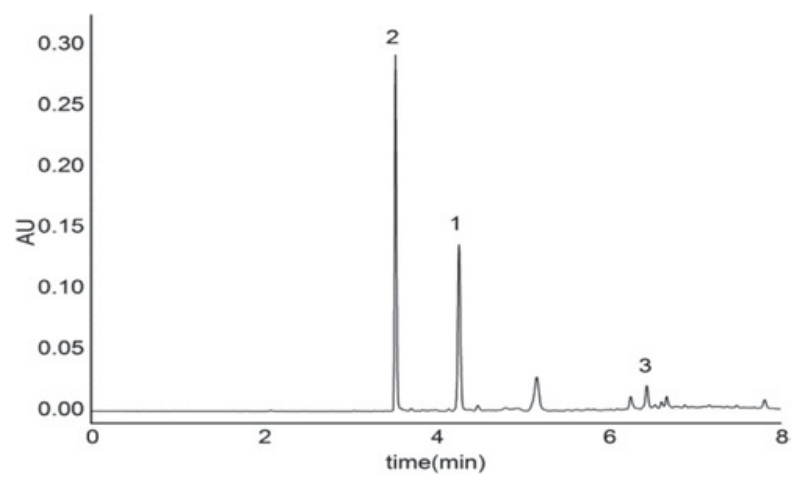

Figure 3. UPLC analysis of fraction I from HSCCC separation: 1(-)-epicatechin $2(+)$-catechin 3 (-)-Epicatechin-3-o-gallate.

monomer catechins and OPCs were first eluted in the tailhead mode with the upper organic phase as the mobile phase and then PPCs were eluted in the head-tail mode with the lower aqueous phase as the mobile phase. As there were no obvious boundaries between OPC and PPC, they could not reach the base line equilibrium. The elution was in the order of the degree of polymerization. The longer the operation time in the tail-head mode with the upper organic phase as the mobile phase, the higher the mDP of the OPC and PPC became. After the tail-head mode with the upper organic phase as the mobile phase for $240 \mathrm{~min}$, the baseline dropped and the loss of the stationary phase sharply increased. In order to ensure the retention rate of the stationary phase, the head-tail mode with the lower aqueous phase as the mobile phase was applied after the tail-head mode for $300 \mathrm{~min}$.

At the flow speed of $2 \mathrm{ml} / \mathrm{min}$, the retention time was too long, OPCs was mainly retained on the column and the fraction II and III were incompletely eluted. While the flow speed was set at $3 \mathrm{ml} / \mathrm{min}$ to improve the elution efficiency, the fraction II, III and IV were eluted. With the 
high flow speed, the low retention rate of the stationary phase affected the separation. The retention rate of the lower aqueous phase as the stationary phase was low to $30 \%$ at the flow speed of $3 \mathrm{ml} / \mathrm{min}$. And the solvent system in the tail-head mode was more unstable than that in the head-tail mode. It was inevitable that the baseline dropped and the loss of the stationary phase sharply increased. Although this limitation, this method could be attempted to fractionate the grape seed crude extracts in the degree of polymerization, which would provide experiment basis for further separation of PAs by HSCCC.

\section{Conclusions}

In this paper, catechins in grape seed crude extracts were a step separation by HSCCC. The developed method is efficient, time-saving and convenient, which can be used for a large-scale isolation of catechins. As well as the work attempted to separate monomer catechins, oligomeric procyandins and polymeric procyandins fractions from grape seed crude extracts according to the polymerization degree. HSCCC has shown to be a great potential for the preparative isolation of the grape crude extracts.

\section{References}

[1] J.M. Yu, M. Ahmedna, Food Sci. \& Technol. 48, 221 (2013)

[2] C. Bladé, L. Arola, M.J.Salvadó, Mol. Nutur. Food Res. 54, 37 (2010)

[3] K.Wang, Z. Liu, J.A. Huang, X. Dong, L. Song, P. Yu, F. liu, J. Chromatogr. B 867, 282 (2008)

[4] S. Bradamante, L. Barenghi, A. Villa, Cardiovasc. Drug Rev. 22, 169 (2004)

[5] J. Ahn, I.U. Grun, L.N. Fernando , J. Food Sci. 67, 1364 (2002)

[6] B.S. Sun, M.C. Leandro, J.M. Ricardo-da-Silva, M.I. Spranger. J Agric. Food Chem. 46, 1390 (1998)

[7] B.S. Sun, G.P. Belchior, J.M. Ricardo-da-Silva, M.I. Spranger, J. Chromatogr. A 841, 115 (1999)

[8] M.I. Spranger, B.S. Sun, A.M. Mateus, V. de Freitas, J.M. Ricardo-da-Silva, Food Chem. 108, 519 (2008)
[9] B.S. Sun, M.I. Spranger. Ciência Téc Vitiv 20, 59 (2005)

[10] X.L. Cao, High-Speed Counter-Current Chromatography Technique and Application, (Chemical Industry Press, Beijing, 2005)

[11] N.S. Kumar, M. Rajapaksha, J. Chromatogr. A 1083, $223(2005)$

[12] G.B. Xia, S.han Hong, S.B. Liu, Food Chem. 151, $380(2014)$

[13] W. Jin, P.F. Tu, J. Chromatogr. A1092, 241 (2005)

[14] E.L. Regalado, S. Tolle, J.A. Pino, P. Winterhalter, R. Menendez, A.R. Morales, J.L. Rodriguez, J. Chromatogr. A 1218, 7358 (2011)

[15] Y. Shibusawa, A. Yanagida, A. Ito, K. Ichihashi, H. Shindo, Y. Ito, J. Chromatogr. A 886, 65 (2000)

[16] D.F. Wu, X.J. Cao, S.H. Wu, J. Chromatogr. A 1223, $53(2012 a)$

[17] Yanagida, A. Shoji, Y. Shibusawa, H. Shindo, M. Tagashira, M. Ikeda, Y. Ito, J. Chromatogr. A, 1112:195 (2006)

[18] A. Yanagida, A.i Shoji, Y. Shibusawa, H. Shindo, M. Tagashira, M. Ikeda, Y. Ito, J. Chromatogr. A1112, 195 (2006)

[19] M. de Sá, V. Justino, M.I. Spranger, Y.Q. Zhao, L. Han, B.S. Sun., Phytochem. Anal. 25, 134 (2014)

[20] B.S. Sun, A.C. Neves, T.A. Fernandes, A.L. Fernandes, N. Mateus, V. de Freitas, C. Leandro, M.I. Spranger, J. Agric. Food Chem. 59, 6550 (2011)

[21] N. Köhler, V. Wray, P. Winterhalter, J. Chromatogr. A1177, 114 (2008)

[22] R. Arimboor, C. Arumughan, J. Food Sci. 77, 1306 (2012)

[23] H.Y. Cui, X.Y. Jia, X. Zhang, J. Zhang, Z.Q. Zhang, Sep. Purif. Technol. 77, 269 (2011)

[24] P. Chen, H.G. Wang, Q.Z. Du, Y. Ito, J. Chromatogr. A $982,163(2002)$

[25] X.L. Cao, C. Wang, H.R. Pei, B.G. Sun, J. Chromatogr. A 1216, 4268 (2009)

[26] Y. Wei, Q.Q. Xie, W.T. Dong, Y. Ito, J. Chromatogr. A 1216, 4313 (2009)

[27] Y. Shibusawa, A. Yanagida, M. Isozaki, H. Shindo, J. Chromatogr. A 915, 253 (2001) 